\title{
Una unidad didáctica intermitente de acondicionamiento físico solo mejora los niveles de capacidad cardiorrespiratoria de los estudiantes con un perfil no saludable de condición física An intermittent physical fitness teaching unit only improves cardiorespiratory fitness levels of students with an unhealthy physical fitness profile \\ *Santiago Guijarro-Romero, **Daniel Mayorga-Vega, *Carolina Casado-Robles, *Jesús Viciana *Universidad de Granada (España), **Universidad de Jaén (España)
}

Resumen. El objetivo del presente estudio fue comparar el efecto de una unidad didáctica intermitente de acondicionamiento físico en Educación Física sobre los niveles de actividad física y capacidad cardiorrespiratoria entre estudiantes con un perfil saludable/no saludable de capacidad cardiorrespiratoria. Ochenta estudiantes de $2^{\circ}-3^{\circ}$ curso de Educación Secundaria fueron asignados aleatoriamente al grupo control y grupo experimental. El grupo experimental, dividido en perfiles saludable/no saludable, realizó una unidad didáctica intermitente de acondicionamiento físico para mejorar la capacidad cardiorrespiratoria. El grupo control trabajó un contenido diferente durante el mismo tiempo y con la misma frecuencia, pero sin hacer hincapié en la mejora de la capacidad cardiorrespiratoria. Antes y después de la intervención, la capacidad cardiorrespiratoria se evaluó objetivamente mediante el test de ida y vuelta de 20 metros. Los niveles de actividad física fueron medidos objetivamente a través de un pulsómetro durante las clases de Educación Física. Ambos perfiles tuvieron mayores niveles de actividad física durante las clases de Educación Física que el grupo control $(p<.001)$. Sin embargo, solo los estudiantes con un perfil no saludable mejoraron significativamente sus niveles de capacidad cardiorrespiratoria tras la unidad didáctica $(p<.01)$. Aunque una unidad didáctica intermitente de acondicionamiento físico parece tener un efecto similar en los niveles de actividad física de todos los perfiles de capacidad cardiorrespiratoria de los estudiantes, solo mejora la capacidad cardiorrespiratoria de los estudiantes con un perfil no saludable.

Palabras clave: Capacidad cardiorrespiratoria, actividad física, intervención educativa, Educación Física, adolescentes, nivel inicial.

\begin{abstract}
The purpose of the present study was to compare the effect of a Physical Education-based physical fitness intermittent teaching unit on physical activity levels and cardiorespiratory fitness among students with healthy/unhealthy cardiorespiratory fitness profile. Eighty students from $2^{\circ}-3^{\circ}$ grades of Secondary Education were randomly assigned to the control group and experimental group. The experimental group, divided into healthy/unhealthy profiles, performed a physical fitness intermittent teaching unit to improve the cardiorespiratory fitness. The control group worked a different content during the same time and with the same frequency, but without emphasizing cardiorespiratory fitness improvement. Before and after the teaching unit, students' cardiorespiratory fitness was objectively measured by the 20-meter shuttle run test. Participants' physical activity levels were measured objectively using a heart rate monitor during Physical Education lessons. Students from both profiles had higher physical activity levels during Physical Education lessons than the control group $(p<.001)$. However, only students with an unhealthy cardiorespiratory fitness profile statistically improved their cardiorespiratory fitness levels after the teaching unit $(p<.01)$. Although an intermittent physical fitness teaching unit seems to have similar effect on physical activity levels of students from all cardiorespiratory fitness profiles, it only improves the cardiorespiratory fitness of those with an unhealthy one.
\end{abstract}

Keywords: Cardiorespiratory fitness, physical activity, educational intervention, Physical Education, adolescents, baseline.

\section{Introducción}

Durante la adolescencia, la actividad física moderadavigorosa (AFMV) está fuerte y positivamente asociada con numerosos marcadores relacionados con la salud como la adiposidad, salud ósea y biomarcadores metabólicos (como el colesterol o la resistencia a la insulina); con la condición física (Poitras, et al., 2016); así como con una mejor salud mental (Biddle, Ciaccioni, Thomas, \& Vergeer, 2018). La Organización Mundial de la Salud recomienda que los adolescentes acumulen diariamente al menos 60 minutos de AFMV (World Health Organization, 2014). Desafortunadamente, aproximadamente el $81 \%$ de los adolescentes a nivel mundial no cumplen esta recomendación (Oviedo, Sánchez, Castro, Calvo, Sevilla, Iglesias, \& Guerra, 2013; World Health Organization, 2014).

El contexto escolar, y específicamente la asignatura de Educación Física (EF), es considerado un entorno clave para la promoción y mejora de los niveles de actividad física (AF) de los estudiantes (Association for Physical Education, 2015),

Fecha recepción: 17-07-19. Fecha de aceptación: 31-10-19

Daniel Mayorga-Vega

dmayorgavega@gmail.com debido a su carácter obligatorio en el currículum educativo y a que es desarrollada por profesionales cualificados (Viciana, Martínez-Baena, \& Mayorga-Vega, 2015). Estudios previos han mostrado que, en los días con $\mathrm{EF}$, los estudiantes acumulan mayores niveles de AFMV que durante los días sin EF (Mayorga-Vega, Martínez-Baena, \& Viciana, 2018; Viciana, Mayorga-Vega, \& Parra-Saldías, 2017). LaAsociación para la EF recomienda que los estudiantes deben estar implicados en AFMV al menos el 50\% del tiempo de las clases de EF (Association for Physical Education, 2015). Sin embargo, en Educación Secundaria casi nunca se cumple esta recomendación (de media, los estudiantes están involucrados el 40.5\% de la clase en AFMV, Hall-López, Ochoa Martínez, Zuñiga Burruel, Monreal Ortiz, \& Sáenz-López Buñuel, 2017; Hollis et al., 2017; Mayorga-Vega, Saldías, \& Viciana, 2020), excepto cuando las clases de EF son específicamente diseñadas para mejorar los niveles de AFMV de los estudiantes (Lonsdale et al., 2013).

La AFMV está positivamente asociada con la condición física de los adolescentes (Marques, Santos, Ekelund, \& Sardinha, 2015). Dentro de la condición física, la capacidad cardiorrespiratoria (CCR) es considerada el marcador de salud más potente en los adolescentes (Tomkinson et al., 2016). Durante la adolescencia, niveles más altos de CCR están 
relacionados con una mejor calidad de vida (Evaristo et al., 2019), así como con mayores niveles de rendimiento académico y cognitivo (Ruíz-Ariza, Grao-Cruces, de Loureiro, \& Martínez-López, 2017). Desafortunadamente, la prevalencia de una baja CCR entre adolescentes es alta a nivel mundial (de media, el 46\% de las mujeres y el 33\% de los hombres), aumentando dicha proporción cada año de edad de media un $10 \%$ en las mujeres y un $8 \%$ en los hombres (Tomkinson et al., 2016). Por lo tanto, es importante que en contextos escolares se promueva y facilite la adquisición de unos niveles saludables de CCR (Tomkinson et al., 2016), siendo esencial la EF en este sentido (Viciana, Mayorga-Vega, \& MerinoMarban, 2014). Sin embargo, aspectos como el gran volumen de contenidos curriculares a desarrollar durante el curso académico, el nivel heterogéneo de los estudiantes, o el tiempo limitado asignado a la asignatura de EF, dificultan conseguir un incremento en los niveles de CCR de los estudiantes (Hardman, Murphy, Routen, \& Tones, 2014). A pesar de estas limitaciones, estudios previos han demostrado que es posible incrementar los niveles de CCR de los estudiantes en la EF (por ejemplo, Mayorga-Vega, Montoro-Escaño, Merino-Marban, \& Viciana, 2016; Mayorga-Vega, Viciana, \& Cocca, 2013).

Sin embargo, debido a la heterogeneidad interna de los grupos educativos (Mayorga-Vega \& Viciana, 2015), las unidades didácticas de condición física aplicadas en los estudios mencionados anteriormente (Mayorga-Vega et al., 2016; Mayorga-Vega et al., 2013), podrían no incrementar en la misma medida los niveles de CCR y AF de todos los estudiantes. Por ejemplo, Resaland, Andersen, Mamen, y Anderssen (2011) demostraron que el efecto de un programa de AF está asociado negativamente con el nivel inicial de CCR. Igualmente, Mayorga-Vega y Viciana (2015) constataron que sólo los estudiantes con un nivel inicial más bajo de CCR mejoraron tras una unidad didáctica de acondicionamiento físico. Sin embargo, estos autores dividieron el grupo experimental según un criterio estadístico (percentil 50) en lugar de dividirlos según un perfil saludable/no saludable de CCR. Además, los autores previos tampoco comprobaron el efecto sobre los niveles de AF de los estudiantes.

Por otro lado, tras las intervenciones escolares mencionadas anteriormente solo se consiguió el objetivo de mejorar la CCR de los estudiantes, teniendo que desarrollar después una gran cantidad de objetivos curriculares en un tiempo limitado (Viciana et al., 2014). En este sentido, Viciana y Mayorga-Vega (2016) proponen diferentes modelos de unidades didácticas innovadoras en función de su distribución temporal, entre la que cabe destacar la unidad didáctica intermitente como una buena solución. Este modelo de unidad didáctica innovadora consiste en trabajar un objetivo curricular solo durante una franja de tiempo de cada clase de EF durante varias clases. Por lo tanto, permitiría dividir la clase de EF en varias partes (por ejemplo, dos), desarrollando dos o más objetivos curriculares en franjas temporales independientes de la clase. Mayorga-Vega et al. (2016) aplicaron este modelo de unidad didáctica para mantener los niveles de CCR previamente adquiridos mediante la realización de actividades de deporte durante solo los primeros 15 minutos de la parte principal de las clases de EF dos veces a la semana durante ocho semanas, mientras el resto de las clases de esta unidad didáctica se destinaron a otros contenidos/objetivos de EF. Igualmente, varios estudios previos han aplicado este modelo de unidad didáctica innovadora para desarrollar y mantener la flexibilidad, utilizando para ello solo el calentamiento o la vuelta a la calma de las clases de EF (Becerra-Fernandez, Merino-Marban, \& Mayorga-Vega, 2016; Mayorga-Vega, Merino-Marban, Redondo-Martín, \& Viciana, 2017; Merino-Marban, Mayorga-Vega, FernandezRodriguez, Estrada, \& Viciana, 2015). Otro ejemplo de unidad didáctica intermitente, podría consistir en desarrollar la CCR de los estudiantes durante solo unos minutos de la parte principal de las clases, dedicando el resto a desarrollar otro objetivo curricular como, por ejemplo, el aprendizaje técnicotáctico de los deportes. Desafortunadamente, no se han encontrado estudios previos analizando el efecto de una unidad didáctica intermitente de estas características sobre el incremento de los niveles de AF y CCR de los estudiantes. Consecuentemente, los objetivos del presente estudio fueron: 1) comparar el efecto de una unidad didáctica intermitente de acondicionamiento físico sobre los niveles de AF durante las clases de EF en estudiantes de Educación Secundaria con un perfil saludable y no saludable de CCR; y 2) comparar el efecto de una unidad didáctica intermitente de acondicionamiento físico sobre los niveles de CCR adquiridos en estudiantes de Educación Secundaria con un perfil saludable y no saludable de CCR.

\section{Material y método}

\section{Participantes}

El protocolo del presente estudio fue aprobado por el Comité Ético de la Universidad de Granada (Referencia: 649/ CEIH/2018). Dos centros públicos de Educación Secundara de la ciudad de Membrilla (Castilla-La Mancha, España) fueron invitados a participar. La muestra inicial estuvo compuesta por un total de 92 estudiantes (34 varones y 58 mujeres) que aceptaron participar en el presente estudio y cumplían los criterios de inclusión. Tras aplicar los criterios de exclusión, 80 estudiantes (32 varones y 48 mujeres) conformaron la muestra final. Los criterios de inclusión fueron: a) estar matriculado en segundo o tercer curso de Educación Secundaria; b) participar en las clases de EF; c) no padecer ninguna enfermedad o lesión que les impidieran realizar AF con normalidad; d) presentar el consentimiento informado firmado por sus padres o tutores legales, y e) presentar el asentimiento informado por parte de los escolares. Los criterios de exclusión fueron: a) no tener un registro válido de todas las variables de estudio (AF y CCR); y b) no tener una tasa de asistencia al programa igual o superior al 85\% durante el periodo de intervención.

Debido a la naturaleza del presente estudio, se empleó un diseño cluster-randomized controlled trial (Campbell \& Stanley, 1963). Las cuatro clases naturales, balanceadas por curso, se asignaron aleatoriamente al grupo control (GC) y grupo experimental (GE). Posteriormente, los estudiantes del GE fueron clasificados en dos subgrupos (saludable (GS) y no saludable (GNS)) de acuerdo con el nivel inicial de CCR (Welk, Laurson, Eisenmann, \& Cureton, 2011). Los participantes del GE tuvieron una asistencia media al programa del $92.6 \%$ (92.4\% y $92.8 \%$ para los subgrupos saludable y no 
saludable, respectivamente). La Tabla 1 muestra las características generales de los participantes incluidos en el presente estudio.

Tabla 1

Características generales de los participantes analizados

\begin{tabular}{|c|c|c|c|c|c|}
\hline & $\begin{array}{c}\text { Total } \\
(n=80)\end{array}$ & $\begin{array}{l}\text { Control } \\
(n=38)\end{array}$ & $\begin{array}{c}\text { No saludable } \\
\quad(n=19)\end{array}$ & $\begin{array}{c}\text { Saludable } \\
(n=23)\end{array}$ & $p^{\mathrm{a}}$ \\
\hline Edad (años) & $13.7(.7)$ & $13.6(.6)$ & $13.9(.8)$ & $13.7(.7)$ & - \\
\hline & & & & 34.8 & 628 \\
\hline nes/mujeres) & $40.0 / 6$ & $31.6 / 6$ & $42.1 /$ & $52.2 /$ & .276 \\
\hline Masa & $.5(11.0)$ & $54.2(9$ & 62.2( & $48.5(6.2) \dagger \dagger$ & $<.001$ \\
\hline Talla (c & $162.1(7.8)$ & $160.8(7.6)$ & $163.7(7.8)$ & $162.8(7.9)$ & .372 \\
\hline Índice c & $20.7(3.6)$ & & & $18.3(1.6)^{* * / \dagger \dagger \dagger}$ & $<.001$ \\
\hline AF extraescolar (horas/ semana) & $2.9(2.0)$ & $2.6(2.0)$ & $2.2(1.9)$ & $4.0(1.8)^{* / \dagger}$ & .003 \\
\hline AF habitual (días/ semana) & $2.7(1.6)$ & $2.4(1.5)$ & $2.3(1.6)$ & $3.5(1.5)^{*} / \dagger$ & . 018 \\
\hline
\end{tabular}
\begin{tabular}{llllll} 
AF habitual (días/ semana) & $2.7(1.6)$ & $2.4(1.5)$ & $2.3(1.6)$ & $3.5(1.5) * / \dagger$ & .018 \\
\hline Nota Datos reportados como media (desviación estándar) 0 porcentaje. $\mathrm{AF}=$ actividad física ${ }^{\text {a Nivel }}$
\end{tabular} de significación del análisis de la varianza de un factor (variables continuas) y de la prueba chicuadrado (variables categóricas). Comparación por pares con el ajuste de Bonferroni: Control vs. No saludable/ Saludable $\left(^{*} p<.05, * * p<.01 \mathrm{y} * * * p<.001\right)$ y No saludable vs. Saludable $(\dagger p<.05, \dagger \dagger p$ $<.01 \mathrm{y} \dagger \dagger$

\section{Medidas}

Antropometría. Las medidas antropométricas se evaluaron al inicio del estudio siguiendo el protocolo de la International Standards for Anthropometric Assessment (Stewart, Marfell-Jones, Olds, \& De Ridder, 2011). Primero se midió la masa corporal (Seca, Ltd., Hamburg, Alemania; precisión $=.1 \mathrm{~kg}$ ) y la talla (Holtain Ltd., Crymmych, Pembs, Reino Unido; precisión $=.1 \mathrm{~cm}$ ), Las medidas se realizaron con los participantes en pantalón corto, camiseta y descalzos. Se realizaron dos mediciones de la masa corporal y la talla, calculándose el promedio de cada una de ellas. Posteriormente, se calculó el índice de masa corporal $\left(\mathrm{kg} / \mathrm{m}^{2}\right)$.

Actividad física extraescolar. Las horas de práctica de AF extraescolar fueron medidas a través de la pregunta enKid (Martínez-Gómez et al., 2009). El cuestionario consiste en una única cuestión: «¿Cuántas horas dedicas a actividades deportivas extraescolares semanales?». Se empleó una escala tipo Likert de 7 puntos que iba de 0 a «Más de $5 »$. El cuestionario enKid ha demostrado una correlación estadísticamente significativa en adolescentes $(r=.43)$ (Martínez-Gómez et al., 2009).

Actividad física habitual. La actividad física habitual fue medida con el cuestionario PACE (Physician-based Assessment and Counseling for Exercise). Este cuestionario consiste en dos preguntas, una que valora cuántos días en la última semana (PACE 1, «En los últimos 7 días, ¿cuántos días hiciste actividad física 60 minutos o más?») y en una semana habitual (PACE 2, «En una semana normal, ¿cuántos días haces actividad física 60 minutos o más?») se realiza al menos 60 minutos de actividad física. El cuestionario PACE ha demostrado una correlación estadísticamente significativa en adolescentes ( $\mathrm{r}=$.43) (Martínez-Gómez et al., 2009).

Actividad física objetiva. La frecuencia cardíaca de los estudiantes fue monitorizada durante las clases de EF mediante pulsómetros (Hellin, Garcia-Jimenez, \& Garcia-Pellicer, 2019; Martinez-López, Moreno-Cerceda, Suarez-Manzano, \& Ruiz-Ariza, 2018; Sarradel, Generelo, \& Zaragoza, 2011; Yuste, García-Jiménez, \& García-Pellicer, 2015) de acuerdo con el protocolo seguido en estudios previos (MayorgaVega et al., 2016). Brevemente, en cada grupo del estudio, se seleccionaron aleatoriamente cinco estudiantes para llevar un monitor de frecuencia cardíaca (Polar RS300X, Finlandia). Todos los estudiantes se midieron dos o tres veces al final del proceso. Se calcularon la frecuencia cardíaca media (latidos por minuto y porcentaje de la frecuencia cardíaca máxi- ma estimada), AF total (porcentaje del tiempo total involucrado en una intensidad igual o superior al 50\% de la frecuencia cardíaca máxima estimada) y AFMV (porcentaje del tiempo total involucrado en una intensidad igual o superior al $70 \%$ de la frecuencia cardíaca máxima estimada). Posteriormente, los estudiantes fueron categorizados según cumpliesen o no la recomendación de conseguir al menos el $50 \%$ de la clase de EF en AFMV (Association for Physical Education, 2015). La frecuencia cardíaca máxima estimada fue calculada utilizando la siguiente ecuación: 209-.7 x edad (años) (Shargarl, Kislev-Cohen, Zigel, Epstein, Pilz-Bursten, \& Tenenbaum, 2015).

Capacidad cardiorrespiratoria. La CCR fue evaluada al principio y al final de la intervención mediante el test de 20 metros ida y vuelta (velocidad inicial de $8.5 \mathrm{~km} / \mathrm{h}$ e incremento de $0.5 \mathrm{~km} / \mathrm{h}$ cada minuto) (Léger, Mercier, Gadoury, \& Lambert, 1988). Todas las mediciones se realizaron bajo las mismas condiciones ambientales, en una instalación deportiva cubierta con un piso no resbaladizo, el mismo día de la semana y en el mismo periodo de tiempo para cada estudiante. Durante el test cada participante llevó puesto un monitor de frecuencia cardíaca (Polar RS300X, Finlandia) para controlar la implicación fisiológica (debiendo ser igual o superior al $90 \%$ de la frecuencia cardíaca máxima). Se registraron el número total de vueltas completadas (n) y el tiempo (en segundos). El volumen máximo de oxígeno (en $\mathrm{ml} / \mathrm{kg} / \mathrm{min}$ ) fue estimado usando la siguiente ecuación: $31.025+3.238 \mathrm{x}$ velocidad $-3.248 \mathrm{x}$ edad $+.1536 \mathrm{x}$ velocidad $\mathrm{x}$ edad (velocidad expresada en $\mathrm{km} / \mathrm{h}$ y edad en el número entero redondeado inferior) (Léger et al., 1988). El test de 20 metros ida y vuelta ha demostrado unos valores adecuados de fiabilidad y validez en adolescentes (por ejemplo, $\mathrm{ICC}=.89 ; r_{\mathrm{p}}=.78$ ) (Léger et al., 1988; Mayorga-Vega, Aguilar-Soto, \& Viciana, 2015).

\section{Procedimiento}

La Figura 1 muestra el esquema general de la intervención. Los estudiantes del GE realizaron una unidad didáctica intermitente de acondicionamiento físico durante las clases de EF dos veces a la semana durante nueve semanas. Finalmente, debido a contingencias educativas tales como días lluviosos, los estudiantes del GE completaron un total de 16 clases. Las clases fueron diseñadas e impartidas por el profesor de EF con la supervisión del investigador principal de acuerdo con el currículum aprobado establecido por el centro.

Cada sesión duró 50 minutos y consistió en cinco a diez minutos de calentamiento, 35-40 minutos de parte principal y cinco minutos de vuelta a la calma. Durante el calentamiento los estudiantes realizaron ejercicios aeróbicos de baja a moderada intensidad seguidos de ejercicios de movilidad articular y estiramientos. Debido a la programación del profesor y con el objetivo de hacer las sesiones más participativas y motivadoras para los estudiantes (Arantes da Costa, Borsato Passos, Matías de Souza, \& Vieira, 2017), la parte principal de las sesiones estuvo dividida en dos mitades de aproximadamente 18-20 minutos cada una. Durante la primera mitad, los estudiantes del GE realizaron ejercicios tradicionales de condición física (por ejemplo, entrenamiento interválico, entrenamiento en circuito, fartlek, juegos de carrera o trabajo con 
combas). Por ejemplo, en la clase con combas los estudiantes realizaban durante diez minutos diferentes saltos a la comba en series de dos minutos y medio con 30 segundos de descanso, seguido del juego de los diez pases por equipos durante diez minutos. Durante la segunda mitad de la clase, los estudiantes del GE trabajaron deportes de invasión (fútbol-sala y baloncesto). Los ejercicios realizados durante esta segunda mitad se caracterizaron por: (a) ser continuos, con las mínimas pausas posibles, y globales; y (b) ser motivantes y basados en situaciones reducidas de juego, tales como el 1x1, 2x1 y 2x3.

El GC realizó dos clases de EF a la semana durante el periodo de intervención y con una estructura similar a la del GE. Sin embargo, la metodología estuvo principalmente basada en la recreación y sin ningún énfasis en la intensidad de la AF. Respecto a los contenidos, el GC trabajó expresión corporal (por ejemplo, ejecución de diferentes figuras de acrosport y montajes con música) y actividades en el medio natural (por ejemplo, juegos de pistas y carreras de orientación).

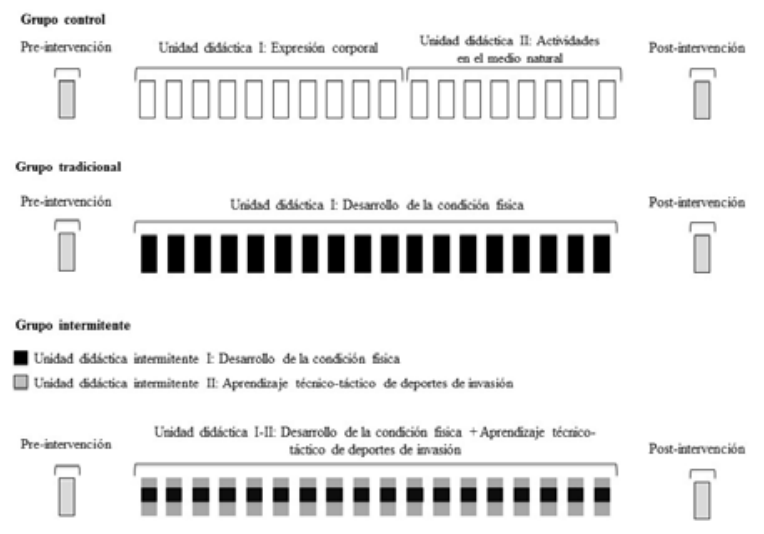

Figura 1. Esquema general de la intervención.

\section{Análisis estadísticos}

Los datos fueron reportados como media (desviación estándar), y mostrados como media (error estándar) en las Figuras. El análisis de la varianza (ANOVA) de un factor (variables continuas) y la prueba de chi-cuadrado (variables categóricas) se utilizaron para examinar diferencias potenciales en términos de características generales entre los estudiantes de los tres grupos. Se utilizó el ANOVA de un factor sobre las variables dependientes [frecuencia cardíaca (ppm), frecuencia cardíaca (\%), AF total, AFMV], incluyendo grupo como variable independiente (GC, GNS, GS) para examinar el efecto de la unidad didáctica intermitente de acondicionamiento físico sobre los niveles de AF. Se utilizó el análisis de covarianza de dos factores (ANCOVA) sobre las variables dependientes [consumo máximo de oxígeno $(\mathrm{ml} /$ $\mathrm{kg} / \mathrm{min}$ ), rendimiento total basado en el tiempo (s)], con edad como covariable, incluyendo grupo como variable independiente (GC, GNS, GS), y tiempo como variable dependiente (pre-intervención, post-intervención) para examinar el efecto de la unidad didáctica intermitente de acondicionamiento físico sobre los niveles de CCR. Posteriormente, las comparaciones por pares se ajustaron con la corrección de Bonferroni. Por otro lado, se calculó la prueba de chi-cuadrado para analizar el efecto de la unidad didáctica sobre el porcentaje de estudiantes que cumplieron la recomendación de AFMV durante las clases de EF entre los tres grupos. Los tamaños del efecto fueron estimados mediante la eta al cuadrado parcial $\left(c^{2}{ }_{\mathrm{p}}\right) / d$ de Cohen y V de Cramer para las variables continuas y dicotómicas, respectivamente (Field, 2017). Todas las asunciones de las pruebas estadísticas aplicadas fueron comprobadas y satisfechas. Todas las covariables potenciales estudiadas (es decir, género, edad, curso, masa corporal, talla, índice de masa corporal, AF extra-escolar, AF habitual, y asistencia al programa) fueron examinadas para ver si debían ser tenidas en cuenta. Todos los análisis estadísticos se realizaron con el programa SPSS, versión 21.0 para Windows (IBM $®$ SPSS $®$ Statistics). El nivel de significación se estableció en valores de $p<.05$, excepto para la prueba $2 \times 2$ chi-cuadrado que fue establecido en $p<.017$.

\section{Resultados}

\section{Actividad física}

La Figura 2 muestra la comparación del efecto de la unidad didáctica intermitente de acondicionamiento físico sobre los niveles de AF en las clases de EF entre los tres grupos. Los resultados del ANOVA de un factor mostraron diferencias significativas entre los tres grupos [frecuencia cardíaca (ppm): $F=25.322 ; p<.001 ; c^{2}=.397$; frecuencia cardíaca (\%): $F=25.357 ; p<.001 ; c^{2}=.397 ; \mathrm{AF}$ total: $F=18.223$; $p<.001 ; c_{\mathrm{p}}^{2}=.321$; AFMV: $\left.F=23.967 ; p<.001 ; \mathrm{c}^{2}=.384\right]$. Posteriormente, las comparaciones por pares mostraron que los estudiantes con un perfil saludable y no saludable de CCR tuvieron estadísticamente mayores niveles de AF durante las clases de EF que los estudiantes del GC [frecuencia cardíaca (ppm), $p<.001, d=1.75$ y 1.46; frecuencia cardíaca $(\%), p<.001, d=1.75$ y $1.44 ;$ AF total $(\%), p<.001, d=1.16$ y $1.01 ; \operatorname{AFMV}(\%), p<.001, d=1.73$ y 1.31$]$. Sin embargo, no se encontraron diferencias entre los estudiantes con un perfil saludable y no saludable [frecuencia cardíaca ( $\mathrm{ppm}), p=$ $1.00, d=.30$; frecuencia cardíaca $(\%), p=.956, d=.31 ; \mathrm{AF}$ total $(\%), p=1.000, d=.14 ; \operatorname{AFMV}(\%), p=.521, d=.42]$.

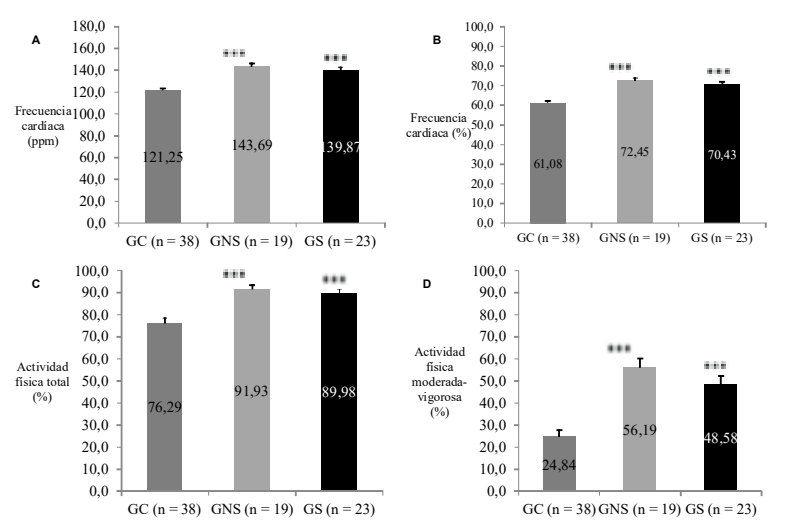

Figura 2. Comparación del efecto de la unidad didáctica intermitente de acondicionamiento físico sobre la frecuencia cardíaca (ppm, A; \%, B), actividad fisica total (\%, C), y niveles de actividad física moderada-vigorosa (\%, D) durante las clases de Educación Física entre los tres grupos. Las barras representan la media y las barras de error representan el error estándar. $\mathrm{GC}=$ Grupo control; GNS = Grupo con un perfil no saludable; GS = Grupo con un perfil saludable. con el ajuste de Bonferroni: GNS/GS vs. GC $\left({ }^{* * *} p<.01\right)$

La Figura 3 muestra la comparación del efecto de la unidad didáctica intermitente de acondicionamiento físico sobre el porcentaje de estudiantes que cumplieron la recomendación de AFMV durante las clases de EF entre los tres 
grupos. Los resultados de la prueba chi-cuadrado sobre el porcentaje de estudiantes cumpliendo la recomendación de AFMV mostraron diferencias estadísticamente significativas entre los tres grupos $\left(\div^{2}=16.254 ; p<.001 ; \mathrm{V}\right.$ de Cramer $=$ .451). Posteriormente, el resultado de la prueba $2 \times 2$ de chicuadrado mostró que hubo un porcentaje mayor de estudiantes con un perfil saludable y no saludable que cumplieron la recomendación de AFMV en clases de EF que en el GC

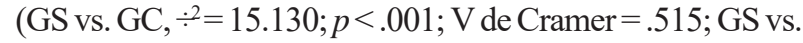
$\mathrm{GC}, \div^{2}=8.900 ; p=.003 ; \mathrm{V}$ de Cramer $\left.=.382\right)$. Sin embargo, no se encontraron diferencias significativas entre los estudian-

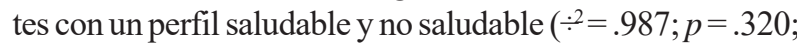
$\mathrm{V}$ de Cramer $=.153$ ).

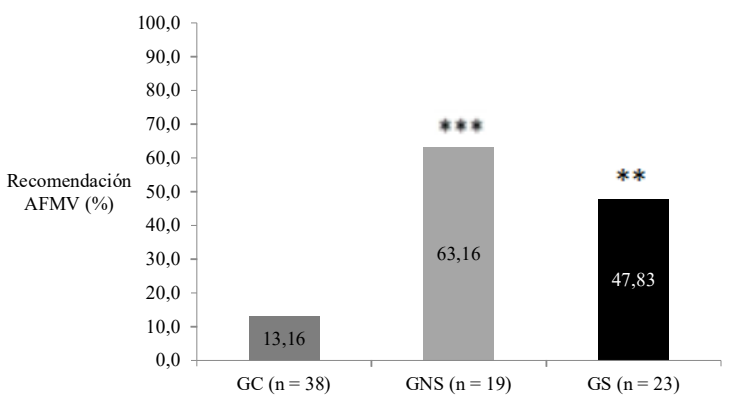

Figura 3. Comparación del efecto de la unidad didáctica intermitente de acondicionamiento físico sobre el porcentaje de estudiantes que cumplieron la recomendación de actividad física moderada-vigorosa (AFMV) durante las clases de Educación Física entre los tres grupos. GC Grupo control; GNS = Grupo con un perfil no saludable; GS = Grupo con un perfil saludable. os resultados del ANOVA de un factor (todas $p<.05$ ) seguido por las comparaciones por pares con el ajuste de Bonferroni: GNS/ GS vs. GC $(* * * p<.001, * * p<.01)$

\section{Capacidad cardiorrespiratoria}

La Figura 4 muestra la comparación del efecto de la unidad didáctica intermitente de acondicionamiento físico sobre los niveles de CCR entre los tres grupos. Los resultados delANCOVAde dos factores mostraron efectos de interacción estadísticamente significativos entre las variables grupo y tiempo para los niveles de CCR [consumo máximo de oxígeno $(\mathrm{ml} / \mathrm{kg} / \mathrm{min}): F=4.842 ; p=.010 ; c_{\mathrm{p}}^{2}=.113$; rendimiento total basado en el tiempo (s): $\left.F=6.338 ; p=.003 ; c^{2}=.143 ;\right]$. Posteriormente, las comparaciones por pares mostraron que los estudiantes con un perfil no saludable de CCR mejoraron de manera estadísticamente significativa sus niveles de CCR en comparación con sus valores iniciales [consumo máximo de oxígeno $(\mathrm{ml} / \mathrm{kg} / \mathrm{min})$, pre-intervención $(M=37.28, D E=$ $1.11)$, post-intervención $(M=38.86, D E=1.12), p=.003, d=$ .32 ; rendimiento total basado en el tiempo (s), pre-intervención $(M=198.58, D E=24.93)$, post-intervención $(M=232.86$, $D E=24.67), p=.001, d=.33]$. Sin embargo, para los estudiantes con un perfil saludable y los estudiantes del GC no se

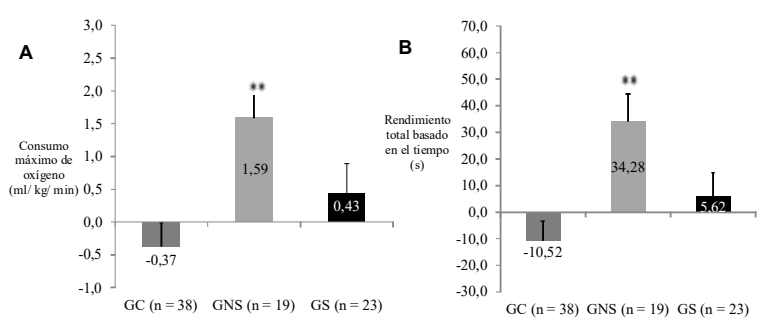

Figura 4. Comparación del efecto de la unidad didáctica intermitente de acondicionamiento físico sobre el volumen máximo de oxígeno $(\mathrm{ml} / \mathrm{kg} / \mathrm{min}$, A) y niveles de rendimiento total basado en el tiempo (s, B) entre los tres grupos. Las barras representan la media ajustada (valor de la diferencia $=$ post-intervención - pre-intervención) y las barras de error representan el error estándar. $\mathrm{GC}=$ Grupo control; GNS $=$ Grupo con un perfil no saludable; $\mathrm{GS}=$ Grupo con un perfil saludable. Los resultados del ANOVA de dos factores (todas $p<.05$ ) seguido por las comparaciones por pares con el ajuste de Bonferroni: ${ }^{* *} p<.01$ encontraron diferencias estadísticamente significativas [consumo máximo de oxígeno $(\mathrm{ml} / \mathrm{kg} / \mathrm{min})$, pre-intervención ( $M$ $=38.91, D E=.78(\mathrm{GC}) ; M=46.59, D E=1.00(\mathrm{GS}))$, postintervención $(M=38.54, D E=.783(\mathrm{GC}) ; M=47.02, D E=1.00$ $(\mathrm{GS})), p>.05, d=.13$; rendimiento total basado en el tiempo (s), pre-intervención $(M=246.47, D E=17.49(\mathrm{GC}) ; M=408.23$, $D E=22.36(\mathrm{GS}))$, post-intervención $(M=235.94, D E=17.32$ $(\mathrm{GC}) ; M=413.85, D E=22.10(\mathrm{GS})), p>.05, d=.12]$.

\section{Discusión}

El primer objetivo del presente estudio fue comparar el efecto de una unidad didáctica intermitente de acondicionamiento físico sobre los niveles de AF durante las clases de EF entre estudiantes de Educación Secundaria con un perfil saludable y no saludable de CCR. Los resultados mostraron que después de la unidad didáctica, los estudiantes con ambos perfiles incrementaron sus niveles de AF durante las clases de EF. De manera similar, Calahorro-Cañada, TorresLuque, López-Fernández, y Carnero (2016) en un estudio descriptivo analizaron las diferencias en los niveles de AF en estudiantes de Educación Secundaria y encontraron que los estudiantes con un perfil saludable de CCR eran más activos que aquellos con un perfil no saludable. Desafortunadamente no se encontraron estudios previos de intervención que comparasen el efecto de una unidad didáctica intermitente de acondicionamiento físico sobre los niveles de AF durante las clases de EF entre estudiantes de Educación Secundaria con un perfil saludable y no saludable de CCR.

Por otro lado, los resultados del presente estudio mostraron que los estudiantes acumularon entre el 50-55\% del tiempo de la clase de EF en AFMV. Estudios previos han mostrado que se puede mejorar hasta en un $24 \%$ el tiempo que los estudiantes están involucrados en AFMV durante las clases de EF (Lonsdale et al., 2013). Sin embargo, el porcentaje del tiempo de la clase de EF dedicado a AFMV es menor que el recomendado (40.5\%) cuando las clases de EF no están específicamente diseñadas para mejorar los niveles de AFMV de los estudiantes (Hollis et al., 2017).

En cuanto a la proporción de estudiantes que cumplieron con la recomendación de acumular al menos el 50\% de las clases de EF en AFMV, los resultados del presente estudio mostraron que el $50 \%$ de los estudiantes con un perfil saludable de CCR y el $60 \%$ de los que tenían un perfil no saludable de CCR, cumplieron dicha recomendación. Sin embargo, la proporción de estudiantes que cumple esta recomendación es prácticamente cero cuando las clases de EF no están específicamente diseñadas para conseguir este objetivo (Viciana et al., 2015). Finalmente, respecto al tamaño del efecto, los resultados del presente estudio $(d=1.31$ hasta 1.73) fueron similares a los de estudios previos centrados en la mejora de los niveles de AFMV durante las clases de EF (.13 hasta 2.81, Lonsdale et al., 2013).

El segundo objetivo fue comparar el efecto de una unidad didáctica intermitente de acondicionamiento físico sobre los niveles de CCR adquiridos entre estudiantes de Educación Secundaria con un perfil saludable y no saludable de CCR. Los resultados del presente estudio mostraron cómo solo los estudiantes con un perfil no saludable de CCR mejoraron sus niveles de CCR después de la unidad didáctica. En 
cambio, los estudiantes con un perfil saludable de CCR no tuvieron incremento. Estudios previos han demostrado que una intervención a corto plazo puede mejorar los niveles de CCR en los adolescentes (por ejemplo, Mayorga-Vega et al., 2016, Mayorga-Vega et al., 2013). Sin embargo, el efecto de una unidad didáctica de condición física está asociado negativamente con el nivel inicial de CCR (Resaland et al., 2011). De manera similar al presente estudio, Mayorga-Vega y Viciana (2015) examinaron el efecto de una unidad didáctica de condición física realizada durante las clases de EF (dos veces por semana) teniendo en cuenta el nivel inicial de CCR de los estudiantes. Estos autores encontraron que solo los estudiantes con un bajo nivel inicial de CCR mejoraron después de la unidad didáctica. Sin embargo, Mayorga-Vega y Viciana (2015) dividieron a los estudiantes del GE estadísticamente por percentiles del nivel inicial de CCR (por debajo del $\mathrm{P}_{50}$ e igual o superior al $\mathrm{P}_{50}$ ), sin tener en cuenta si los niveles de CCR eran saludables o no. Por consiguiente, las mejoras obtenidas por los estudiantes pudieron no ser suficientes para adquirir unos niveles saludables de CCR, ya que inicialmente fueron clasificados por percentiles en lugar de en función de un criterio de referencia relacionado con la salud (Welk et al., 2011). Igualmente, Resaland et al. (2011) observaron que la aplicación de un programa de AF diario durante dos años tuvo mayor impacto en los estudiantes con un nivel inicial bajo de CCR. Sin embargo, la frecuencia y duración total de este programa fue totalmente diferente al tiempo designado para la asignatura de EF en la mayoría de los países (dos horas a la semana) (Hardman et al., 2014).

Por otro lado, en los estudios mencionados anteriormente que aplicaron una unidad didáctica de acondicionamiento físico a corto plazo (Mayorga-Vega et al., 2016, MayorgaVega \& Viciana, 2015; Mayorga-Vega et al., 2013), solo consiguieron mejorar la CCR de los estudiantes, teniendo que desarrollar después otros objetivos curriculares en un tiempo limitado (Hardman et al., 2014). En este sentido, otro resultado importante del presente estudio, es que además de mejorar los niveles de CCR de los estudiantes con un perfil de CCR no saludable, al mismo tiempo, se trabajaron otros objetivos curriculares como aspectos técnico-tácticos de deportes de invasión (fútbol-sala y baloncesto).

Respecto a la magnitud del efecto, en el presente estudio el tamaño del efecto fue moderado $(d=.32)$ para los estudiantes con un perfil de CCR no saludable y muy bajo $(d=$ .13) para los estudiantes con un perfil saludable de CCR. De manera similar, Mayorga-Vega y Viciana (2015) encontraron también un efecto moderado para los estudiantes con menor nivel de CCR $(d=.44)$ y muy bajo para aquellos que tenían mayor nivel de CCR $(d=.07)$. Posiblemente, la razón por la que los estudiantes con un perfil saludable de CCR no mejoraron sus niveles de CCR después de la intervención, pudo ser que las clases realizadas no fuesen estímulo suficiente debido a la baja frecuencia semanal de la asignatura de EF en España, similar a la mayoría de los países de nuestro entorno (Hardman et al., 2014).

Las fortalezas de este estudio fueron: 1) es el primer estudio que analiza el efecto de una unidad didáctica intermitente de acondicionamiento físico dividiendo a los estudiantes por perfiles de acuerdo a su nivel inicial de consumo máximo de oxígeno (Welk et al., 2011), en lugar de por un criterio estadístico como en el estudio de Mayorga-Vega y Viciana (2015); 2) muestra el efecto en los niveles medios de AF diferenciando por perfiles de CCR; y 3 ) el porcentaje de estudiantes que cumplieron la recomendación de AFMV durante las clases de EF. Respecto a las limitaciones, la principal limitación del estudio fue que el aprendizaje técnico-táctico no fue medido debido a problemas de viabilidad. En España, el tiempo destinado a Educación Física es limitado (solo dos horas a la semana) y el año académico es interrumpido por las vacaciones aproximadamente cada tres meses. Por lo tanto, dado que la evaluación objetiva de aprendizaje técnicotáctico habría necesitado más sesiones de evaluación, no se pudo realizar. En segundo lugar, el consumo máximo de oxígeno fue estimado a través de una ecuación validada, ya que su medición directa no era viable por razones prácticas. Estudios futuros, podrían medir el consumo máximo de oxígeno de forma directa con un analizador de gases portátil con el fin de obtener una medida objetiva de este parámetro.

\section{Aplicaciones prácticas}

La asignatura de EF tiene un enorme potencial sobre los estudiantes para la adquisición de los 60 minutos diarios de AFMV (Mayorga-Vega, Martínez-Baena, \& Viciana, 2018). Por tanto, conseguir que los alumnos estén involucrados al menos el 50\% del tiempo de la clase de EF en AFMV (Association for Physical Education, 2015) resulta un aspecto clave. Planificar unidades didácticas intermitentes de acondicionamiento físico a través de ejercicios tradicionales de condición física y de deportes colectivos, podría ser una buena herramienta para los profesores de EF con el fin de aumentar los niveles de AFMV durante sus clases. Además de mejorar los niveles de AFMV durante las clases de EF, este tipo de unidades didácticas permitirían solucionar los problemas de planificación presentes en la asignatura de EF a la hora de desarrollar una cualidad física tan importante en la adolescencia como la capacidad cardiorrespiratoria (Hardman et al., 2014; Viciana et al., 2014). Incluso, con objeto de mejorar esta cualidad física en ambos perfiles de estudiantes (saludable/no saludable), los profesores de EF podrían planificar unidades didácticas intermitentes de acondicionamiento físico estableciendo diferentes niveles de intensidad dentro de la misma clase. De esta forma, se conseguiría un estímulo de trabajo suficiente para mejorar esta cualidad física en todos los estudiantes.

\section{Conclusiones}

De lo que conocemos este es el primer estudio que compara el efecto de una unidad didáctica intermitente de acondicionamiento físico sobre los niveles de CCR adquiridos y los niveles de AF durante las clases de EF entre estudiantes de Educación Secundaria con un perfil saludable y no saludable de CCR. Ambos perfiles de estudiantes tuvieron mayores niveles de AFMV tras la aplicación de la unidad didáctica intermitente. Además, hubo un mayor porcentaje de alumnos en ambos perfiles que cumplieron con la recomendación de AFMV durante las clases de EF. Sin embargo, una unidad didáctica intermitente de acondicionamiento físico de dos sesiones por semana solo mejora la CCR de los estudiantes 
con un perfil no saludable de CCR. Por lo tanto, con el propósito de incrementar la CCR de todos los estudiantes, parece necesario aumentar la carga lectiva de la asignatura de EF, por ejemplo, mediante un aumento de la frecuencia semanal.

\section{Agradecimientos}

Agradecemos a todos los alumnos y padres su participación voluntaria e incondicional en el presente estudio. Agradecemos también a todos los miembros del centro escolar su entusiasmo y colaboración, especialmente al equipo directivo y profesores del departamento de Educación Física. Se recibe una ayuda del Ministerio de Ciencia, Innovación y Universidades del Gobierno de España (Santiago Guijarro-Romero: FPU15/02387; Carolina Casado-Robles: FPU16/03314).

\section{Referencias}

Arantes da Costa, L. C., Borsato Passos, P. C., Matías de Souza, V. F., \& Vieira, L. F. (2017). Physical Education and sports: Motivation for school daily practice. Movimento, 23(3), 935-947. doi:10.22456/1982-8918.66430

Association for Physical Education. (2015). Health Position Paper. Worcester: Association for Physical Education.

Becerra-Fernandez, C. A., Merino-Marban, R., \& MayorgaVega, D. (2016). Effect of a physical education-based dynamic stretching program on hamstring extensibility in female high-school students. Kinesiology, 48(2), 258266.

Biddle, S. J. H., Ciaccioni, S., Thomas, G, \& Vergeer, I. (2018). Physical activity and mental health in children and adolescents: An updated review of reviews and an analysis of causality. Psychology of Sport and Exercise, 42, 146-155. doi:10.1016/j.psychsport.2018.08.011

Calahorro-Cañada, F., Torres-Luque, G., López-Fernández, I., \& Carnero, E. A. (2016). Is physical education an effective way to increase physical activity in children with lower cardiorespiratory fitness? Scandinavian Journal of Medicine and Science in Sports, 27(11), 1417-1422. doi:10.1111/sms.12740

Campbell, D. T., \& Stanley, J. C. (1963). Experimental and quasi-experimental designs for research. Boston: Houghton Mifflin Company.

Evaristo, S., Moreira, C., Lopes, L., Oliveira, A., Abreu, S., Agostinis-Sobrinho, C., ... Mota, J. (2019). Muscular fitness and cardiorespiratory fitness are associated with health-related quality of life: Results from labmed physical activity study. Journal of Exercise Science and Fitness, 17(2), 55-61. doi:10.1016/j.jesf.2019.01.002

Field, A. (2017). Discovering statistics using IBM SPSS Statistics $\left(5^{\text {th }}\right.$ ed.). London: SAGE Publications.

Hardman, K., Murphy, C., Routen, A., \& Tones, S. (2014). UNESCO-NWCPEA: World-wide survey of school Physical Education. París: United Nations Educational, Scientific and Cultural Organization.

Hall-López, J., Ochoa Martínez, P., Zuñiga Burruel, R., Monreal Ortiz, L., \& Sáenz-López Buñuel, P. (2017). Moderate-tovigorous physical activity during recess and physical education among mexican elementary school students.
Retos, 31, 137-139.

Hellin, M., Garcia-Jimenez, J. V., \& Garcia-Pellicer, J. J. (2019). Intensity of physical education lessons in children according to the type of activity: Soccer, badminton, aerobics and motor skills. Journal of Physical Education and Sport, 19(1), 603-610. doi:10.7752/jpes.2019.01088

Hollis, J. L., Sutherland, R., Williams, A. J., Campbell, E., Nathan, N., Wolfenden, L., ... Wiggers, J. (2017). A systematic review and meta-analysis of moderate-tovigorous physical activity levels in secondary school physical education lessons. International Journal of Behavioral Nutrition and Physical Activity, 14(1), 52. doi:10.1186/s12966-017-0504-0

Léger, L. A., Mercier, D., Gadoury, C., \& Lambert, J. (1988). The multistage 20 metre shuttle run test for aerobic fitness. Journal of Sports Sciences, 6(2), 93-101. doi:10.1080/ 02640418808729800

Lonsdale, C., Rosenkranz, R. R., Peralta, L. R., Bennie, A., Fahey, P., \& Lubans, D. R. (2013). A systematic review and meta-analysis of interventions designed to increase moderate-to-vigorous physical activity in school physical education lessons. Preventive Medicine, 56(2), 152-161. doi:10.1016/j.ypmed.2012.12.004

Marques, A., Santos, R., Ekelund, U., \& Sardinha, L. B. (2015). Association between physical activity, sedentary time, and healthy fitness in youth. Medicine and Science in Sports and Exercise, 47(3), 575-580. doi:10.1249/ MSS.0000000000000426

Martínez-Gómez, D., Martínez-De-Haro, V., Del-Campo, J., Zapatera, B., Welk, G. J., Villagra,A., .. Veiga, Ó. L. (2009). Validez de cuatro cuestionarios para valorar la actividad física en adolescentes españoles. Gaceta Sanitaria, 23(6), 512-517. doi:10.1016/j.gaceta.2009.02.013

Martinez-López, E. J., Moreno-Cerceda, J., Suarez-Manzano, S., \& Ruiz-Ariza, A. (2018). Efecto y satisfacción de un programa de actividad física controlada por pulsómetros en el índice de masa corporal de escolares con sobrepesoobesidad. Retos, 2041(33), 179-184.

Mayorga-Vega, D., Aguilar-Soto, P., \& Viciana, J. (2015). Criterion-related validity of the 20-m shuttle run test for estimating cardiorespiratory fitness: A meta-analysis. Journal of Sports Science and Medicine, 14(3), 536547. doi:10.1016/j.jad.2014.11.061

Mayorga-Vega, D., Martínez-Baena,A., \& Viciana, J. (2018). Does school physical education really contribute to accelerometer-measured daily physical activity and non sedentary behaviour in high school students? Journal of Sports Sciences, 36(17), 1913-1922. doi:10.1080/ 02640414.2018.1425967

Mayorga-Vega, D., Merino-Marban, R., Redondo-Martín, F. J., \& Viciana, J. (2017). Effect of a one-session-per-week physical education-based stretching program on hamsting extensibility in schoolchildren. Kinesiology, 49(1), 101-108.

Mayorga-Vega, D., Montoro-Escaño, J., Merino-Marban, R., \& Viciana, J. (2016). Effects of a physical education-based programme on health-related physical fitness and its maintenance in high school students: A clusterrandomized controlled trial. European Physical Education Review, 22(2), 243-259. doi:10.1177/ 


\section{$1356336 X 15599010$}

Mayorga-Vega, D., Saldías, M. P., \& Viciana, J. (2020). Niveles objetivos de actividad física durante las clases de Educación Física en estudiantes chilenos usando acelerometría. Retos, 37, 123-128.

Mayorga-Vega, D., \& Viciana, J. (2015). Las clases de educación física solo mejoran la capacidad cardiorrespiratoria de los alumnos con menor condición física: Un estudio de intervención controlado. Nutricion Hospitalaria, 32(1), 330-335. doi:10.3305/nh.2015.32.1.8919

Mayorga-Vega, D., Viciana, J., \& Cocca, A. (2013). Effects of a circuit training program on muscular and cardiovascular endurance and their maintenance in schoolchildren. Journal of Human Kinetics, 37(37), 153-160. doi:10.2478/ hukin-2013-0036

Merino-Marban, R., Mayorga-Vega, D., FernandezRodriguez, E., Estrada, F. V., \& Viciana, J. (2015). Effect of a physical education-based stretching programme on sitand-reach score and its posterior reduction in elementary schoolchildren. European Physical Education Review, 21(1), 83-92. doi:10.1177/1356336X14550942

Oviedo, G., Sánchez, J., Castro, R., Calvo, M., Sevilla, J. C., Iglesias, A., \& Guerra, M. (2013). Niveles de actividad física en población adolescente: estudio de caso. Retos, 23, 43-47.

Poitras, V., Gray, C., Borghese, M., Carson, V., Chaput, J., Janssen, I., ... Tremblay, M. (2016). Systematic review of the relationships between objectively measured physical activity and health indicators in school-aged children and youth. Applied Physiology, Nutrition, and Metabolism, 41(6), 197-239. doi:10.1139/apnm-2015-0663

Resaland, G. K., Andersen, L. B., Mamen,A., \& Anderssen, S. A. (2011). Effects of a 2-year school-based daily physical activity intervention on cardiorespiratory fitness: The Sogndal school-intervention study. Scandinavian Journal of Medicine and Science in Sports, 21(2), 302309. doi:10.1111/j.1600-0838.2009.01028.x

Ruíz-Ariza, A., Grao-Cruces, A., de Loureiro, N. E. M., \& Martínez-López, E. J. (2017). Influence of physical fitness on cognitive and academic performance in adolescents: A systematic review from 2005-2015. International Review of Sport and Exercise Psychology, 10(1), 108133. doi:10.1080/1750984X.2016.1184699

Sarradel, J., Generelo, E., \& Zaragoza, J. (2011). Gender differences in heart rate responses to different types of physical activity in Physical Education classes. European Journal of Human Movement, 26(1), 65-76.

Shargarl, E., Kislev-Cohen, R., Zigel, L., Epstein, S., PilzBursten, R., \& Tenenbaum, G. (2015). Aged-related maximal heart rate: examination refinement of prediction equations. The Journal of Sports Medicine and Physical Fitness, 55(1), 1207-1218. doi:10.1002/ 14651858.CD003793.pub2.7.

Stewart, A., Marfell-Jones, M., Olds, T., \& De Ridder, J. (2011). International standards for anthropometric assessment. New Zealand: International Society for the Advancement of Kinanthropometry.

Tomkinson, G. R., Lang, J. J., Tremblay, M. S., Dale, M., Leblanc, A. G., Belanger, K., ... Léger, L. (2016). International normative $20 \mathrm{~m}$ shuttle run values from 1142026 children and youth representing 50 countries. British Journal of Sports Medicine, 51(21), 1545-1554. doi:10.1136/ bjsports-2016-095987

Viciana, J., Martínez-Baena,A., \& Mayorga-Vega, D. (2015). Contribución de la educación física a las recomendaciones diarias de actividad física en adolescentes según el género; un estudio con acelerometría. Nutricion Hospitalaria, 32(3), 1246-1251. doi:10.3305/nh.2015.32.3.9363

Viciana, J., \& Mayorga-Vega, D. (2016). Innovative teaching units applied to Physical Education - changing the curriculum management for authentic outcomes. Kinesiology, 48(1), 142-152.

Viciana, J., Mayorga-Vega, D., \& Merino-Marban, R. (2014). Physical education-based planning for developing and maintaining students' health-related physical fitness levels. In R. Todaro (Ed.), Handbook of physical education research. Role of school programs, childrens attitudes and health implications (pp. 237-252). New York: Nova Science Publisher.

Viciana, J., Mayorga-Vega, D., \& Parra-Saldías, M. (2017). Adolescents' physical activity levels on physical education and non-physical education days according to gender, age, and weight status. European Physical Education Review, 25(1), 143-155. doi:10.1177/ $1356336 X 17706683$

Welk, G., Laurson, K., Eisenmann, J., \& Cureton, K. (2011). Development of youth aerobic-capacity standards using receiver operating characteristic curves. American Journal of Preventive Medicine, 41(4), 111-116. doi:10.1016/j.amepre.2011.07.007

World Health Organization. (2014). Global status report on noncommunicable diseases 2014. Geneva: World Health Organization.

Yuste, J. L., García-Jiménez, J. V., \& García-Pellicer, J. J. (2015). Intensidad de las clases de Educación Física en adolescentes. Revista Internacional de Medicina y Ciencias de la Actividad Fì̀sica y el Deporte, 15(58), 309-323.

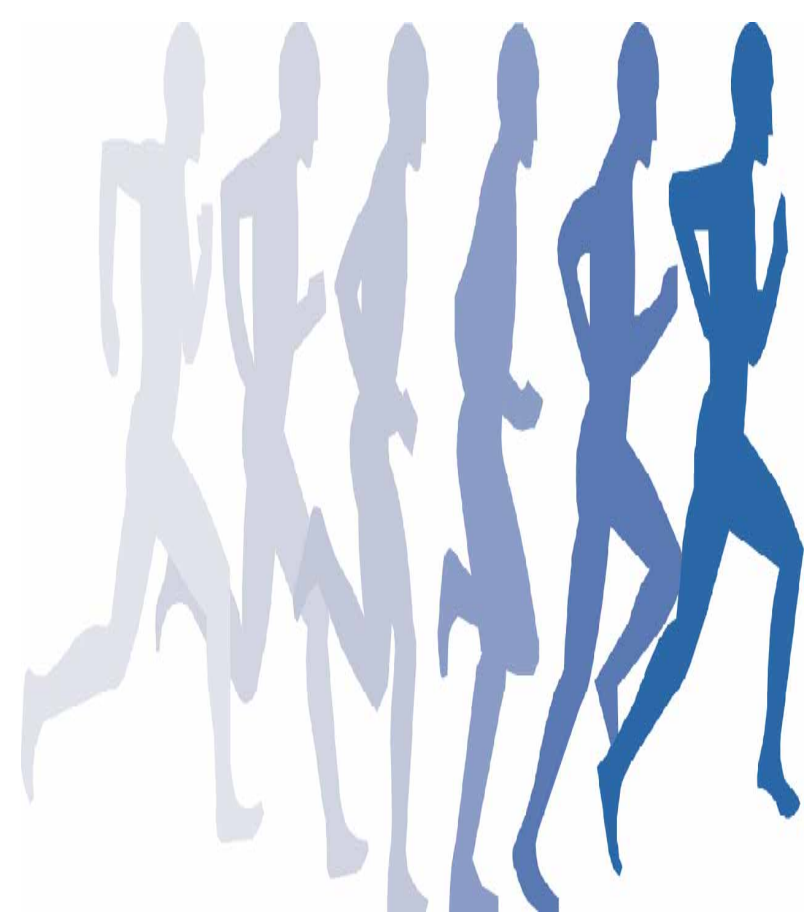

